\title{
Intrapartum asphyxia and hypoxic ischaemic encephalopathy in a public hospital: Incidence and predictors of poor outcome
}

\author{
E K Bruckmann, MB ChB; $\boldsymbol{S}$ Velaphi, MB ChB, FCPaed \\ Department of Paediatrics, Faculty of Health Sciences, University of the Witwatersrand, Johannesburg, South Africa, and \\ Chris Hani Baragwanath Academic Hospital, Johannesburg
}

Corresponding author: E Bruckmann (eduard.bruckmann@wits.ac.za)

\begin{abstract}
Objective. To determine the incidence of asphyxia and hypoxic ischaemic encephalopathy (HIE) and predictors of poor outcome in a hospital in a developing country.

Methods. Neonates of birth weight $\geq 2000 \mathrm{~g}$ who required bag-and-mask ventilation and were admitted with a primary diagnosis of asphyxia from January to December 2011 were included. Medical records were retrieved and maternal and infant data collected and analysed. Infants who had severe HIE and/or died were compared with those who survived to hospital discharge with no or mild to moderate HIE.

Results. There were 21086 liveborn infants with a birth weight of $\geq 2000 \mathrm{~g}$ over the study period. The incidence of asphyxia ranged from 8.7 to $15.2 / 1000$ live births and that of HIE from 8.5 to 13.3/1 000, based on the definition of asphyxia used. In $60 \%$ of patients with HIE it was moderate to severe. The overall mortality rate was $7.8 \%$. The mortality rate in infants with moderate and severe HIE was $7.1 \%$ and $62.5 \%$, respectively. The odds of severe HIE and/or death were high if the Apgar score was $<5$ at 10 minutes (odds ratio (OR) $19.1 ; 95 \%$ confidence interval (CI) 5.7 - 66.9) and if there was no spontaneous respiration at 20 minutes (OR 27.2; 95\% CI 6.9 - 117.4), a need for adrenaline (OR 81.2; 95\% CI 13.2 - 647.7) and a pH of <7 (OR 5.33; 95\% CI 1.31 - 25.16). Predictors of poor outcome were Apgar score at 10 minutes $(p=0.004)$, need for adrenaline $(p=0.034)$ and low serum bicarbonate $(p=0.028)$.

Conclusion. The incidence of asphyxia in term and near-term infants is higher than that reported in developed countries. Apgar score at 10 minutes and need for adrenaline remain important factors in predicting poor outcome in infants with asphyxia.

S Afr Med J 2015;105(4):298-303. DOI:10.7196/SAMJ.9140
\end{abstract}

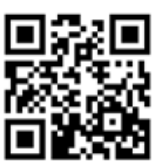

Asphyxia is defined biochemically as a condition of impaired respiratory gaseous exchange that leads to hypoxaemia, hypercapnia and metabolic acidosis. Metabolic acidaemia, defined as a base deficit of $>12 \mathrm{mmol} / \mathrm{L}$, has been associated with episodes of intrapartum asphyxia and has therefore been used to define asphyxia biochemically. ${ }^{[1]}$ The problem with this definition is that a number of normal babies have metabolic acidosis, and if it is used on its own there is a risk that the incidence of asphyxia may be overestimated. ${ }^{[2]}$ Clinically, the need for bag-and-mask ventilation (BMV) at birth and/or an Apgar score $<7$ have been used to define intrapartum asphyxia. ${ }^{[3]}$ Since in many developing countries there are no facilities to do blood gas measurement, base deficit is not used as part of the definition and only the clinical definition is used. Using the clinical definition alone to describe the incidence of asphyxia may also overestimate the incidence, as infants who do not initiate respiration are not always asphyxiated intrapartum. Assessing the incidence of asphyxia using a combination of clinical definition of need for BMV, Apgar score $<7$ at 5 minutes and metabolic acidaemia (base deficit $>12 \mathrm{mmol} / \mathrm{L}$ ) is therefore more likely to provide information on the true incidence of asphyxia in any population. Neonates with asphyxia often have ongoing respiratory depression requiring respiratory support in a neonatal intensive care unit (NICU). Where resources are limited, there is competition for these interventions, resulting in rationing and their being offered to infants who are more likely to survive with minimal morbidity. It is therefore important to determine factors that are associated with severe morbidity and mortality, as they could assist in decision-making on who should be offered these limited resources.
In this study we sought to assess the incidence of asphyxia and hypoxic ischaemic encephalopathy (HIE) according to the presence of signs that define HIE 1, 2 and 3 in Sarnat staging (Table 1), ${ }^{[4]}$ infant characteristics and factors associated with severe HIE and mortality in term and near-term neonates diagnosed with asphyxia in a setting where there is a high patient load and resources are limited.

\section{Methods}

Study design. A retrospective, descriptive study of neonates born at term or near term (defined as birth weight $\geq 2000 \mathrm{~g}$ ) and admitted with a diagnosis of asphyxia.

Study setting. Chris Hani Baragwanath Academic Hospital (CHBAH), Johannesburg, South Africa, a public government hospital. This hospital serves the population of Soweto and the surrounding areas. Until April 2014, it was the only hospital in Soweto. It is also a referral centre for all clinics conducting births in Soweto and surrounding areas. These clinics conduct about 8000 births per year and the hospital about 23000 births per year, with a total of just over 30000 births per year for the cluster. In 2011, the hospital had two operating theatres for obstetric surgery including caesarean sections (CSs), and 12 NICU beds.

Study population. Infants who were born weighing $\geq 2000 \mathrm{~g}$, required resuscitation with at least BMV and were admitted with a diagnosis of asphyxia were included. The study period was from 1 January to 31 December 2011. Infants who died in the labour ward soon after birth were excluded, as their records were either missing or had incomplete information. Infants born before arrival to hospital, twins and infants with severe congenital abnormalities were also excluded. 
Table 1. Sarnat and Sarnat ${ }^{[4]}$ classification of HIE

\begin{tabular}{|c|c|c|c|}
\hline & Stage 1 & Stage 2 & Stage 3 \\
\hline Level of consciousness & Hyperalert & Lethargic or obtunded & Stuporous \\
\hline \multicolumn{4}{|l|}{ Neuromuscular control } \\
\hline Muscle tone & Normal & Mild hypotonia & Flaccid \\
\hline Posture & Mild distal flexion & Strong distal flexion & Intermittent decerebration \\
\hline Stretch reflexes & Overactive & Overactive & Decreased or absent \\
\hline Segmental myoclonus & Present & Present & Absent \\
\hline \multicolumn{4}{|l|}{ Complex reflexes } \\
\hline Suck & Weak & Weak or absent & Absent \\
\hline Moro & Strong; low threshold & $\begin{array}{l}\text { Weak; incomplete; high } \\
\text { threshold }\end{array}$ & Absent \\
\hline Oculovestibular & Normal & Overactive & Weak or absent \\
\hline Tonic neck & Slight & Strong & Absent \\
\hline Autonomic function & Generalised sympathetic & Generalised parasympathetic & Both systems depressed \\
\hline Pupils & Mydriasis & Miosis & Variable; often unequal; poor light reflex \\
\hline Heart rate & Tachycardia & Bradycardia & Variable \\
\hline Bronchial and salivary secretions & Sparse & Profuse & Variable \\
\hline Gastrointestinal motility & Normal or decreased & Increased; diarrhoea & Variable \\
\hline Seizures & None & Common; focal or multifocal & Uncommon (excluding decerebration) \\
\hline
\end{tabular}

\section{Table 2. Incidence of asphyxia according to different definitions of asphyxia ( $N=21086$ live births)}

\begin{tabular}{lll}
\hline Definition & Infants with asphyxia, $\boldsymbol{n}$ & Incidence /1 000 live births \\
\hline Need for BMV at birth & 321 & 15.2 \\
BMV + base deficit $>12 \mathrm{mmol} / \mathrm{L}$ & 261 & 12.4 \\
$\mathrm{BMV}+$ Apgar score $<7$ at $5 \mathrm{~min}$ & 218 & 10.3 \\
BMV, Apgar score $<7$ at $5 \mathrm{~min}+$ & 183 & 8.7 \\
base deficit $>12 \mathrm{mmol} / \mathrm{L}$ & &
\end{tabular}

Study procedure. Hospital records of newborn infants who met the inclusion criteria were retrieved and the following data were collected: birth weight, gender, gestational age, growth, Apgar score, resuscitation required, time to spontaneous respiration, arterial blood gas done within the first hour of birth, and the diagnosis of HIE. Maternal records were also reviewed for maternal age, parity, maternal HIV results, antenatal care, maternal disease, mode of delivery, presence of fetal distress or meconium-stained amniotic fluid and cardiotocographic records. Means, standard deviations (SDs), medians and ranges were used to describe continuous variables, while frequencies and percentages were used to describe categorical variables. The $\chi^{2}$ test or Fisher's exact test and Student's $t$-test were used to compare the categorical variables and continuous variables, respectively, between infants with no or mild to moderate HIE and those with severe HIE or who died. Differences between the two groups were considered statistically significant at a $p$-value of $<0.05$.
The extent of association of different variables with severe HIE or death was reported using odds ratios (ORs) and the precision using 95\% confidence intervals (CIs). In order to determine predictors of severe HIE or death, variables with $p$-values $<0.05$ on the univariate logistic regression were included in the multivariate logistic regression. STATA version 10.0. was used to perform the statistical analysis.

Approval to conduct the study was obtained from the hospital protocol review committee and the University of the Witwatersrand Human Research Ethics Committee.

\section{Results}

\section{Incidence of asphyxia}

Of a total of 23035 liveborn infants, 21086 weighed $\geq 2000 \mathrm{~g}$ at birth. Of those weighing $\geq 2000 \mathrm{~g}, 357 \mathrm{had}$ a diagnosis of asphyxia, of whom 321 were admitted and 36 died in the labour ward before admission; since records of the latter were either missing or had incomplete information, they were excluded.
The incidence of asphyxia ranged from 8.7 to $15.2 / 1000$ deliveries, depending on the definition of asphyxia used (Table 2). Using the definition combining need for BMV, Apgar score $<7$ at 5 minutes and base deficit $>12 \mathrm{mmol} / \mathrm{L}$ (representing a truer reflection of intrapartum asphyxia) the incidence was $8.7 / 1000$ live births, compared with 15.2/1 000 using need for BMV alone.

\section{Maternal characteristics}

Demographic and clinical characteristics of mothers giving birth to infants diagnosed with asphyxia are shown in Table 3. Most of the mothers (95.6\%) had attended antenatal care. The average maternal age was just over 25 years, and $57.6 \%$ were pregnant for the first time. Just over a quarter of mothers (25.9\%) were positive for HIV. Among the mothers who had maternal illness recorded, the most common diagnosis was pregnancyinduced hypertension, accounting for $75.9 \%$ of mothers with recorded illness and $19.6 \%$ of mothers giving birth to infants with a diagnosis of asphyxia. Of babies with asphyxia, $34.3 \%$ were born to mothers with meconium-stained amniotic fluid. The mode of delivery was CS in $38.0 \%$ of cases, with only $8.7 \%$ of deliveries being assisted vaginal deliveries. The most common reason for performing CS in mothers of asphyxiated infants was fetal distress (68.9\%) followed by prolonged second stage of labour (7.3\%), cephalopelvic disproportion (6.3\%) and cord prolapse (4.1\%). Electronic monitoring with a 
Table 3. Characteristics of mothers who gave birth to infants with asphyxia $(N=321)$

\begin{tabular}{|c|c|}
\hline Variables & \\
\hline Maternal age (years), mean (SD) & $25.17(6.4)$ \\
\hline First pregnancies, $n(\%)$ & $185(57.6)$ \\
\hline Positive HIV test, $n$ (\%) & $83(25.9)$ \\
\hline Received antenatal care, $n$ (\%) & 307 (95.6) \\
\hline \multicolumn{2}{|l|}{ Maternal illness, $n(\%)$} \\
\hline Hypertension & $63(19.6)$ \\
\hline Other medical conditions & $20(6.2)$ \\
\hline \multicolumn{2}{|l|}{ Mode of delivery, $n(\%)$} \\
\hline CS & $122(38.0)$ \\
\hline Vaginal delivery & $171(43.2)$ \\
\hline Assisted vaginal delivery & $28(8.7)$ \\
\hline Meconium-stained amniotic fluid, $n(\%)$ & $110(34.3)$ \\
\hline \multicolumn{2}{|c|}{$\begin{array}{l}\text { Documented electronic monitoring during labour (CTG), } \\
n(\%)\end{array}$} \\
\hline Yes & $249(77.6)$ \\
\hline No & $72(22.4)$ \\
\hline Normal CTG in monitored patients, $n$ (\%) & $132 / 249(53)$ \\
\hline Abnormal CTG in monitored patients, $n$ (\%) & $117 / 249(47)$ \\
\hline Late decelerations & $90 / 117(76.9)$ \\
\hline Early decelerations & $8 / 117(6.8)$ \\
\hline Fetal bradycardia & $8 / 117(6.8)$ \\
\hline Beat-to-beat variability & 3/117 (2.6) \\
\hline Suspicious tracing & $8 / 117(6.8)$ \\
\hline
\end{tabular}

cardiotocograph (CTG) was performed in $77.6 \%$ of cases; tracings were normal in $53 \%$ and abnormal in $47 \%$. In mothers with an abnormal CTG, the common abnormality was late deceleration (76.9\%), followed by early deceleration $(6.8 \%)$ and fetal bradycardia $(6.8 \%)$.

\section{Infant characteristics (Table 4)} Apgar scores

The average birth weight was $3084 \mathrm{~g}$ and the average gestation 38.5 weeks. Apgar scores were done at 1 and 5 minutes in $99.5 \%$ of the infants and at 10 minutes in $72.6 \%$. At 5 minutes $68.0 \%$ had an Apgar score of $<7$. Of infants with a documented Apgar score at 10 minutes, $29.7 \%$ had a score of $<7$.

\section{Resuscitation and time to spontaneous respiration}

During resuscitation, the majority $(89.7 \%)$ of infants diagnosed with asphyxia responded to BMV only, while just over $10 \%$ required extensive resuscitation in the form of chest compressions (7.2\%) and adrenaline (3.1\%). Of the infants who had time to spontaneous respiration recorded, $50.0 \%$ took $>5$ minutes to attain spontaneous respiration.

\section{Blood gases}

All infants were resuscitated with $100 \%$ oxygen. The majority of infants had blood gas analysis performed within an hour after delivery. The mean partial pressure of oxygen $\left(\mathrm{PaO}_{2}\right)$ and partial pressure of carbon dioxide $\left(\mathrm{PaCO}_{2}\right)$ were $145.6 \mathrm{mmHg}$ and 36.6 $\mathrm{mmHg}$, respectively, suggesting overzealous BMV. Only $24.0 \%$ of infants had a $\mathrm{pH}$ of $<7.00,57.9 \%$ had a $\mathrm{PaO}_{2}$ of $>100 \mathrm{mmHg}, 53.0 \%$
Table 4. Characteristics of infants diagnosed with asphyxia $(N=321)$

\section{Variables}

Birth weight (g), mean (SD)

3084 (448)

Gestational age (weeks), mean (SD)

$38.5(2.2)$

Gender, $n(\%)$

Male

$192(59.8)$

Female

$129(40.2)$

Apgar score at $5 \mathrm{~min}, n(\%)$

$<5$

$75(23.4)$

$5-7$

$143(44.6)$

$\geq 7$

$101(31.5)$

Apgar score at $10 \mathrm{~min}, n(\%)$

Recorded $233(72.6)$

$<5$

$19 / 233(8.2)$

$5-7$

$50 / 233(21.5)$

$\geq 7$

$164 / 233(70.3)$

Not recorded, $n(\%)$

88 (27.4)

Resuscitation required, $n$ (\%)

BMV only

$288(89.7)$

$\mathrm{BMV}+\mathrm{CC}$

$\mathrm{BMV}+\mathrm{CC}+$ adrenaline

$10(3.1)$

Time to spontaneous breathing (min), $n$ (\%)

$<5$

105 (32.7)

$5-10$

89 (27.7)

$10-20$

$42(13.1)$

$>20$

27 (8.4)

Not recorded

58 (18.1)

$\mathrm{pH}$, mean (SD)

$7.09(0.16)$

$\mathrm{pH}<7.00, n(\%)$

77 (24.0)

pH $7.00-7.25, n(\%)$

$182(56.7)$

$\mathrm{pH}>7.25, n(\%)$

38 (11.8)

$\mathrm{PaO}_{2}(\mathrm{mmHg})$, mean (SD)

$145.63(79.92)$

$\mathrm{PaO}_{2}<50, n(\%)$

16 (5)

$\mathrm{PaO}_{2} 50-100, n$ (\%)

92 (28.7)

$\mathrm{PaO}_{2}>100, n(\%)$

$186(57.9)$

$\mathrm{PaCO}_{2}(\mathrm{mmHg})$, mean (SD)

$36.66(16.71)$

$\mathrm{PaCO}_{2}<35, n(\%)$

$170(53.0)$

$\mathrm{PaCO}_{2} 35-45, n(\%)$

64 (19.9)

Base deficit (mmol/L), mean (SD)

$18.09(5.26)$

Base deficit $\leq 12, n(\%)$

35 (10.9)

Base deficit $>12, n(\%)$

$261(81.3)$

$\mathrm{CC}=$ chest compressions.

had hypocarbia $\left(\mathrm{PaCO}_{2}<35 \mathrm{mmHg}\right)$, and $81.3 \%$ had a base deficit of $>12 \mathrm{mmol} / \mathrm{L}$. The lactate level, which is a good indicator of tissue hypoxia, was found to be $>7.5 \mathrm{mmol} / \mathrm{L}$ in $97.4 \%$ of cases in which it was documented.

\section{Incidence of HIE and mortality}

The incidence of HIE ranged from 8.5 to $13.3 / 1000$ live births, depending on the definition of asphyxia used (Table 5). Defining 
asphyxia as an Apgar score of $<7$ at 5 minutes and base deficit of $>12 \mathrm{mmol} / \mathrm{L}, 179$ patients (97.8\%) developed signs of HIE (according to Sarnat and Sarnat staging ${ }^{[4]}$, giving an incidence of 8.5/1 000 live births, compared with 13.3/1 000 live births when asphyxia was defined as need for BMV. Among those who developed HIE, 59.0\% had moderate to severe HIE. The overall mortality rate was $7.8 \%$, with the rates in infants with HIE ranging from $8.9 \%$ to $12.3 \%$, depending on the definition of asphyxia. Only $1.4 \%$ of infants with HIE 1 died, as opposed to $7.1 \%$ and $62.5 \%$ of those with HIE 2 and 3 respectively, defining asphyxia with inclusion of metabolic acidaemia. There were no deaths among those who did not develop HIE. Sixty-four per cent of the deaths were primarily due to HIE, and $16.0 \%$ were due to HIE and meconium aspiration syndrome, with or without persistent pulmonary hypertension of the newborn; $24.0 \%$ were due to sepsis. Comorbidities in patients with asphyxia included renal dysfunction in $31.2 \%$, clinically presumed sepsis in $24.6 \%$ and positive blood culture in $13.9 \%$.

\section{Comparison of mild to moderate \\ HIE with severe HIE and/or death}

Comparison was made between the above two groups for all patients with asphyxia defined as need for BMV. On univariate analysis, there were no statistically significant differences in maternal characteristics and CTG readings between those with no HIE or mild to moderate HIE compared with those with severe HIE or who died. The infant factors that were associated with poor outcome (severe HIE or death) were low Apgar scores at 5 and 10 minutes, need for adrenaline, delay in time to spontaneous respiration, low $\mathrm{pH}$, high base deficit and low bicarbonate. The median Apgar score was lower at 5 minutes ( 4 v. $6 ; p<0.01)$ and 10 minutes (5 v. $8 ; p<0.01)$, time to spontaneous respiration was longer (15.4 minutes v. 6.2

Table 5. Incidence of HIE and mortality rate in patients with asphyxia, depending on definition used

\begin{tabular}{|c|c|c|c|c|c|c|}
\hline & \multicolumn{3}{|c|}{ Need for BMV } & \multicolumn{3}{|c|}{ Apgar score $<7+$ base deficit $>12 \mathrm{mmol} / \mathrm{L}$} \\
\hline & $\begin{array}{l}\text { Total } \\
N(\%)\end{array}$ & $\begin{array}{l}\text { Incidence } \\
\text { /1 } 000 \text { live } \\
\text { births }\end{array}$ & $\begin{array}{l}\text { Died } \\
n(\%)\end{array}$ & $\begin{array}{l}\text { Total } \\
N(\%)\end{array}$ & $\begin{array}{l}\text { Incidence } \\
\text { /1 } 000 \text { live } \\
\text { births }\end{array}$ & $\begin{array}{l}\text { Died } \\
n(\%)\end{array}$ \\
\hline No HIE & $40(12.5)$ & - & $0(0)$ & $4(2.2)$ & - & $0(0)$ \\
\hline HIE 1 & $131(40.8)$ & 6.2 & $2(1.5)$ & $71(38.8)$ & 3.4 & $1(1.4)$ \\
\hline HIE 2 & $122(38.0)$ & 5.8 & $7(5.7)$ & $84(45.9)$ & 4.0 & $6(7.1)$ \\
\hline HIE 3 & $28(8.7)$ & 1.3 & $16(57.1)$ & $24(13.1)$ & 1.1 & $15(62.5)$ \\
\hline All HIEs & $281(87.5)$ & 13.3 & $25(8.9)$ & $179(97.8)$ & 8.5 & $22(12.3)$ \\
\hline
\end{tabular}

minutes; $p<0.01), \mathrm{pH}$ was lower (6.9 v. 7.1 ; $p<0.01)$, base excess was higher $(22.8 \mathrm{v}$. $17.5 \mathrm{mmol} / \mathrm{L})$ and bicarbonate was lower $(8.9$ v. $11.6 \mathrm{mmol} / \mathrm{L})$ in infants with severe HIE and/or who died compared with those with either no HIE or mild to moderate HIE who survived to hospital discharge (Table 6). Factors showing statistically significant differences in univariate analysis were included in a multivariate analysis to determine factors that could predict severity of HIE or death. Only Apgar score at 10 minutes $(p=0.004)$, need for adrenaline $(p=0.034)$ and bicarbonate levels in the first hour after birth $(p=0.02)$ were found to be predictors of severe HIE and/or death.

\section{Severity of different variables and odds of developing severe HIE or death}

The odds of having severe HIE or death increased if the Apgar score at 5 minutes was <5 (OR 9.33; 95\% CI 2.83 - 34.03); if the Apgar score at 10 minutes was 5 - 7 (OR 3.05; 95\% CI 1.08 - 8.62) or $<5$ (OR 19.1; 95\% CI 5.66 - 66.9) compared with a score of $>7$; if chest compressions were required (OR 4.51; 95\% CI 1.06 - 18.0); if adrenaline was required (OR 81.2; 95\% CI 13.2 - 647.7); and if time to spontaneous respiration was $>20$ minutes (OR 27.2; 95\% CI 6.89 - 117.4). When arterial blood gas was measured within the first hour of life, the odds of having severe HIE and/or death were increased if the $\mathrm{pH}$ was $<7$ (OR 5.33; 95\% CI 1.31 - 25.16); if the $\mathrm{PaO}_{2}$ was $<60 \mathrm{mmHg}$ (OR 9.65; 95\% CI 1.87 - 55.12); and if the $\mathrm{PaCO}_{2}$ was $>45 \mathrm{mmHg}$ (OR 3.98; 95\% CI 1.11 - 15.56) (Table 7).

Table 6. Factors associated with severe HIE or death in infants with asphyxia

\begin{tabular}{|c|c|c|c|c|}
\hline & \multirow{2}{*}{$\begin{array}{l}\text { No HIE or HIE } 1-2 \\
(N=256)\end{array}$} & \multirow{2}{*}{$\begin{array}{l}\text { HIE } 3 \text { and/or death } \\
(N=37)\end{array}$} & \multicolumn{2}{|c|}{$p$-value } \\
\hline & & & Univariate & Multivariate \\
\hline Median Apgar at $5 \mathrm{~min}$ & 6 & 4 & $<0.001$ & 0.99 \\
\hline Median Apgar at $10 \mathrm{~min}$ & 8 & 5 & $<0.001$ & 0.004 \\
\hline Need for CCs, $n(\%)$ & $19 / 256(7.4)$ & $4 / 37(10.8)$ & 0.36 & N/A \\
\hline Need for adrenaline, $n(\%)$ & $1 / 256(0.4)$ & $9 / 37(24.3)$ & $<0.001$ & 0.03 \\
\hline Time to spontaneous respiration (min), mean (SD) & $6.17(5.71)$ & $15.36(11.93)$ & $<0.001$ & 0.69 \\
\hline $\mathrm{pH}$, mean $(\mathrm{SD})$ & $7.11(0.14)$ & $6.92(0.18)$ & $<0.001$ & 0.54 \\
\hline $\mathrm{PaO}_{2}(\mathrm{mmHg})$, mean $(\mathrm{SD})$ & $145.25(76.00)$ & $148.75(108.35)$ & 0.82 & N/A \\
\hline $\mathrm{PaCO}_{2}(\mathrm{mmHg})$, mean $(\mathrm{SD})$ & $34.94(13.89)$ & $49.91(27.69)$ & $<0.001$ & 0.33 \\
\hline Bicarbonate (mmol/L), mean (SD) & $11.59(2.84)$ & $8.91(2.72)$ & $<0.001$ & 0.03 \\
\hline Base deficit (mmol/L), mean (SD) & $17.48(4.88)$ & $22.74(5.79)$ & $<0.001$ & 0.30 \\
\hline Lactate $(\mathrm{mmol} / \mathrm{L})$, mean $(\mathrm{SD})$ & $15.90(4.34)$ & $18.26(3.68)$ & 0.008 & 0.61 \\
\hline Glucose (mmol/L), mean (SD) & $6.79(2.30)$ & $6.93(4.48)$ & 0.82 & N/A \\
\hline
\end{tabular}


Table 7. ORs for developing severe hypoxic ischaemic encephalopathy and/or death according to severity of different variables

\begin{tabular}{|c|c|}
\hline Variables & OR $(95 \% \mathrm{CI})$ \\
\hline \multicolumn{2}{|c|}{ Apgar score at $5 \mathrm{~min}$} \\
\hline$>7$ & Reference \\
\hline $5-7$ & $1.79(0.50-7.01)$ \\
\hline$<5$ & $9.33(2.83-34.03)$ \\
\hline \multicolumn{2}{|c|}{ Apgar score at $10 \mathrm{~min}$} \\
\hline$>7$ & Reference \\
\hline $5-7$ & $3.05(1.08-8.62)$ \\
\hline$<5$ & $19.13(5.66-66.89)$ \\
\hline \multicolumn{2}{|c|}{ Extent of resuscitation } \\
\hline BMV only & Reference \\
\hline CCs & $4.51(1.06-17.98)$ \\
\hline Adrenaline & $81.2(13.17-647.7)$ \\
\hline \multicolumn{2}{|c|}{ Time to spontaneous respiration (min) } \\
\hline$<5$ & Reference \\
\hline $5-10$ & $2.18(0.55-9.24)$ \\
\hline $10-20$ & $3.41(0.74-16.20)$ \\
\hline$>20$ & $27.19(6.89-117.38)$ \\
\hline \multicolumn{2}{|l|}{$\mathrm{pH}$} \\
\hline$>7.25$ & Reference \\
\hline $7.0-7.2$ & $1.48(0.35-7.13)$ \\
\hline$<7.0$ & $5.33(1.31-25.16)$ \\
\hline \multicolumn{2}{|l|}{$\mathrm{PaO}_{2}(\mathrm{mmHg})$} \\
\hline $60-100$ & Reference \\
\hline$>100$ & $2.40(0.63-10.74)$ \\
\hline$<60$ & $9.65(1.87-55.12)$ \\
\hline \multicolumn{2}{|l|}{$\mathrm{PaCO}_{2}(\mathrm{mmHg})$} \\
\hline $35-45$ & Reference \\
\hline$>45$ & $3.98(1.11-15.56)$ \\
\hline$<35$ & $0.75(0.19-3.07)$ \\
\hline
\end{tabular}

\section{Discussion}

The recent development of induced hypothermia having been reported to reduce the risk of mortality and disability in infants with asphyxia prompted this study to assess the burden of asphyxia (incidence of asphyxia) and the numbers of infants who might require cooling (incidence of moderate to severe HIE) in a setting where there is a high patient load but limited resources. Defining asphyxia as need for BMV, Apgar score $<7$ at 5 minutes and base deficit $>12 \mathrm{mmol} / \mathrm{L}$ within an hour after delivery, the incidence of asphyxia was 8.7/1 000 live births. Most studies reporting on the incidence of birth asphyxia in developing countries do not include blood gases, and use only need for assistance with respiration and/or Apgar score $<7$ at 5 minutes. Using the definition of need for assistance with breathing or Apgar score $<7$ at 5 minutes, our incidence of asphyxia was 15.2/1 000 or $10.3 / 1000$ live births, respectively. Whichever definition is used, the incidence of asphyxia in this study is very high compared with that reported in developed countries $\left(\sim 1-5 / 1000\right.$ live births. ${ }^{[5,6]}$ We did not collect data on modifiable factors that may have contributed to this high incidence. A perinatal care survey conducted in South Africa in 2008/9 reported that delay in seeking medical care, inadequate monitoring during labour and lack of facilities were modifiable factors identified in neonates who died from asphyxia. ${ }^{[7]}$ We postulate that these same factors contribute to our high incidence of asphyxia.

The overall incidence of encephalopathy in infants with Apgar scores $<7$ and base deficit $>12 \mathrm{mmol} / \mathrm{L}$ was $8.5 / 1000$ live births, with $60 \%$ of cases being due to HIE 2 and 3 . The overall incidence reported in this study was higher than the incidences reported from Sweden, a developed country, and Kathmandu in Nepal, a developing country (1.8 and 6.4/1 000 live births, respectively ${ }^{[8.9]}$ ), but lower than the 28.1/1 000 reported for Sarlahi, Nepal. ${ }^{[10]}$

It has been reported that of infants diagnosed with HIE, 15 - 20\% will die in the neonatal period and $25-30 \%$ of survivors will develop permanent neurodevelopmental abnormalities including cerebral palsy ${ }^{[6]}$ In our study, $62 \%$ and $7 \%$ of infants with HIE 3 and HIE 2, respectively, died before hospital discharge. Some infants with HIE 2 and most of those with HIE 3 who survive to hospital discharge will have a poor neurological outcome.

The high incidence of HIE in this report is a major concern and highlights the need for improvements in the healthcare system to reduce the incidence of asphyxia and HIE. It is also important to treat infants with moderate to severe HIE with induced hypothermia to reduce neurological disability and mortality.

Predictors of severe HIE and/or death were low Apgar score at 10 minutes, need for adrenaline and low bicarbonate. Shah et al. ${ }^{[11]}$ reported similar findings, and that need for chest compressions, high base deficit and delay in onset of respiration beyond 20 minutes in infants with asphyxia were associated with poor outcome; rates of severe adverse effects were $64 \%$ if one predictor was present, $76 \%$ if two were present and $93 \%$ if all three were present. The finding that Apgar score at 10 minutes is a predictor of poor outcome is helpful, as the Apgar score is easy to perform and widely used in labour and delivery rooms in both developed and developing countries. Although the Apgar score has not been helpful in predicting which infants will develop cerebral palsy, especially in the era of induced hypothermia for asphyxia, ${ }^{[12]}$ it has been shown to be very useful in predicting survival in both preterm and term infants. Laptook et al.$^{[13]}$ reported that each point decrease in Apgar score at 10 minutes was associated with a $45 \%$ increase in the odds of death or disability, and concluded that Apgar score at 10 minutes provided useful prognostic information before other evaluations are available.

The presence of metabolic acidaemia or low bicarbonate is of additional value to the Apgar score in predicting poor outcome, as the Apgar score is often not done in real time and can therefore be unreliable. A base deficit of $>16 \mathrm{mmol} / \mathrm{L}$ has previously been reported to be a predictor of poor outcome. ${ }^{[10]}$ Casey et al.$^{[14]}$ also reported that a combination of low Apgar score $(0-3)$ and cord $\mathrm{pH}$ of $<7.0$ increased the risk of mortality in both preterm and term infants, whereas Sehdev $e t$ al. ${ }^{[15]}$ reported that a base deficit of $>16$ and a 5-minute Apgar score of $<7$ together identified $79 \%$ of neonates who would develop complications.

\section{Study limitations}

The chief limitation of this study is that it is retrospective. The diagnoses of asphyxia and encephalopathy were therefore mainly based on what was recorded in the patient's hospital file. This may have led to overestimation of the true incidence of HIE, especially as some clinical findings could be affected by the experience of a person conducting the examination, e.g. junior doctors commonly labelling infants with asphyxia as having HIE. Our study nevertheless serves to highlight the burden of asphyxia and encephalopathy at $\mathrm{CHBAH}$, which possibly reflects the incidence in other public sector hospitals in South Africa. 


\section{Conclusion}

The incidences of both asphyxia and HIE are very high at CHBAH. As moderate to severe HIE is likely to be associated with abnormal neurodevelopmental outcomes and disability, factors that contribute to a high incidence of asphyxia must be avoided. Infants at risk of developing encephalopathy must be offered neuroprotective treatment, i.e. induced hypothermia. Where arterial blood gas analysis, NICU facilities and induced hypothermia are not available or are limited, an Apgar score of $<5$ at 10 minutes and the need for adrenaline can be of assistance in predicting poor outcome.

\section{References}

1. Low JA, Lindsay BG, Derrick EJ. Threshold of metabolic acidosis associated with newborn complications. Am J Obstet Gynecol 1997;177(6):1391-1394. [http://dx.doi.org/10.1016/S0002 9378(97)70080-2]

2. Josten BE, Johnson TR, Nelson JP. Umbilical cord blood pH and Apgar scores as an index of neonatal health. Am J Obstet Gynecol 1987;157(4):843-848. [http://dx.doi.org/10.1016/S0002-9378(87)80069-8] 3. Ersdal HL, Mduma E, Svensen E, Perlman J. Birth asphyxia: A major cause of early neonatal mortality in a Tanzanian rural hospital. Pediatrics 2012;129(5):e1238-el243. [http://dx.doi.org/10.1542/ peds.2011-3134]

4. Sarnat HB, Sarnat MS. Neonatal encephalopathy following fetal distress: A clinical and electroencephalographic study. Arch Neurol 1976;33(10):696-705. [http://dx.doi.org/10.1001/
[ archneur. 1976.00500100030012

5. Wu YW, Backstrand KH, Zhao S, Fullerton HJ, Johnston SC. Declining diagnosis of birth asphyxia in California: 1991-2000. Pediatrics 2004;114(6):1584-1590. [http://dx.doi.org/10.1542/peds.2004-0708]
6. Tomashek KM, Crouse CJ, Iyasu S, Johnson CH, Flowers LM. A comparison of morbidity rates attributable to conditions originating in the perinatal period among newborns discharged from United States hospitals, 1989-90 and 1999-2000. Paediatr Perinat Epidemiol 2006;20(1):24-34. [http://dx.doi.
org/10.1111/j.1365-3016.2006.00690.x]

2008-2009: Seventh Report on Perinatal Care in South Africa. Pretoria: Tshepesa Press, 2011:28.

. Thiringer $\mathrm{K}$, Odeback A, Milsom I. Birth asphyxia: Incidence, clinical course and outcome in a Swedish population. Acta Paediatr 1995;84(8):927-932. [http://dx.doi. org/10.1111/j.1651-2227.1995.tb13794.x]

9. Ellis M, Manandhar N, Manandhar DS, Costello AM. Risk factors for neonatal encephalopathy in Kathmandu, Nepal, a developing country: Unmatched case-control study. BMJ 2000;320(7244):12291236. [http://dx.doi.org/10.1136/bmj.320.7244.1229]

10. Lee AC, Mullany LC, Tielsch JM, et al. Incidence of and risk factors for neonatal respiratory depression and encephalopathy in rural Sarlahi, Nepal. Pediatrics 2011;128(4):e915-24 [http://dx.doi.org/10.1542/ peds.2010-3590]

11. Shah PS, Beyene J, To T, Ohlsson A, Perlman M. Postasphyxial hypoxic-ischemic encephalopathy in neonates: Outcome prediction rule within 4 hours of birth. Arch Pediatr Adolesc Med 2006;160(7):729736. [http://dx.doi.org/10.1001/archpedi.160.7.729]

12. Apgar V. A proposal for a new method of evaluation of the newborn infant. Curr Res Anesth Analg 1953;32(4):260-267.

13. Laptook AR, Shankaran S, Ambalavanan N, et al. Outcome of term infants using Apgar scores at 10 minutes following hypoxic-ischemic encephalopathy. Pediatrics 2009;124(6):1619-26 [http://dx.doi. org/10.1542/peds.2009-0934]

14. Casey BM, McIntire DD, Leveno KJ. The continuing value of the Apgar score for the assessment of newborn infants. N Engl J Med 2001;344(7):467-471. [http://dx.doi.org/10.1056/ NEJM20010215344070

15. Sehdev HM, Stamilio DM, Macones GA, Graham E, Morgan MA. Predictive factors for neonatal morbidity in neonates with an umbilical arterial cord $\mathrm{pH}$ less than 7.00. Am J Obstet Gynecol 1997;177(5):1030-1034. [http://dx.doi.org/10.1016/S0002-9378(97)70008-5]

Accepted 13 February 2015. 